\section{Nasopharyngeal Swab Collec- tion Methods for COVID-19: Suggestions from COVID-19 Diagnosticians}

Sir,

COVID-19 pandemic challenged the diagnosticians due to massive testing for SARS-CoV-2 in various clinical and epidemiological contexts. So far, nasopharyngeal swabs are the preferred specimen type due to their robust detection for COVID-19. Nasopharyngeal swab collection sites and personnel were the bottlenecks, for which strategies were adopted, among which, notable was the design of less-invasive swabs, ${ }^{1}$ where only mid-turbinate area of nasal passage is reached, alleviating the need for personal protective equipment.

The techniques for nasopharyngeal swab collection for COVID-19 vary, yet are critical to the diagnosis. These involve putting a flexible swab through one of the nostrils to a depth of 7 centimeters. However, there is minimal guidance for optimal swab collection and the duration for the swab to be left in place. The World Health Organization and the US Centers for Disease Control and Prevention $(C D C)^{1,2}$ advised that the swab be left in place for few seconds and gently rubbed and rolled before withdrawal. However, it is uncomfortable; and better understanding of swab collection may enhance patient's cooperation and improve the collection technique.

The observations made in the literature to improve the nasopharyngeal swab collection methods are related to nasal anatomy and the handling of the flexible swab. ${ }^{1-3}$ It was suggested not to apply force while swabbing, and take the sample from mid-turbinate area of the nasal cavity, in case of nasal obstruction (e.g. nasal polyps or deviated nasal septum). ${ }^{1-3}$ Swab saturation is accomplished by a brief placement in the nasopharynx; hence, rotational swab movements are not required. ${ }^{4}$ It does not improve the sample quality. Swabbing from both nostrils is also considered unnecessary and does not recruit additional nucleic acid on swabs. ${ }^{4}$ Such observations help swab collectors to make it a priority to review and standardised the techniques to reduce discomfort level faced by many. There is an interest in alternative options for nasopharyngeal sampling for COVID-19 testing, such as the self-administration of these swabs, used by large-s- cale institutions. These nasal swabs are easy to use and function as point-of-care testing. ${ }^{4}$ They are useful in the settings of businesses and universities to halt the local spread. However, there are limitations associated with diagnostic sensitivity; hence, large-scale testing is needed to confirm their utility.

\section{PATIENT'S CONSENT:}

Notapplicable.

\section{CONFLICT OF INTEREST:}

The author declared no conflict of interest.

\section{AUTHOR'S CONTRIBUTION:}

SS: Solely drafted the manuscript.

\section{REFERENCES}

1. Kinloch NN, Ritchie G, Brumme CJ, Dong W, Lawson T, Jones $\mathrm{RB}$, et al. Suboptimal biological sampling as a probable cause of false-negative COVID-19 diagnostic test results. J Infect Dis 2020; 222(6):899-902. doi: 10.1093/infdis/ jiaa370.

2. World Health Organization. WHO Guidelines for the collection of human specimens for laboratory diagnosis of avian influenza infection. Available at: www.who.int/ influenza/ human_animal_interface/virology_laboratories_ and_vaccines/guidelines_collection_h5n1_humans/en/.

3. US Centers for Disease Control and Prevention. Interim guidelines for collecting, handling and testing clinical specimens for COVID-19. www.cdc.gov/coronavirus/ 2019ncov/lab/guidelines-clinical speci mens.html? CDC_AA refVal=http\%3A\%2F\%2Fwww.cdc.gov\%2Fcoronavirus\% $\overline{2} \mathrm{~F} \overline{2}$ 019ncov\%2Fguidelines-clinical-specimens.html.

4. Marty FM, Chen K, Verrill KA. How to obtain a nasopharyngeal swab specimen. Reply N Engl J Med 2020; 382 (22):e14. doi: 10.1056/NEJMvem2010260.

\section{Sadaf Sheikh}

Sultan Qaboos University Hospital, Muscat, Oman

Correspondence to: Dr. Sadaf Sheikh, Department of Emergency Medicine, Sultan Qaboos University Hospital, Muscat, Oman

E-mail: sheikh.sadaf@gmail.com

Received: March 15, 2021; Revised: June 07, 2021;

Accepted: June 13, 2021

DOI: https://doi.org/10.29271/jcpsp.2021.JCPSPCR.CR146 